NOTA

\title{
Nidada atípica de tero común (Vanellus c. chilensis y V. C. lampronotus, Aves: Charadriidae] en Neuquén y Buenos Aires, Argentina
}

Atypical Clutch of the Southern Lapwing (Vanellus c. chilensis and V. C. lampronotus, Birds: Charadriidae) in Neuquén and Buenos Aires, Argentina

\author{
Mauro Bianchini \\ Independencia 1424, Neuquén capital, (8300) Neuquén. Argentina. \\ Correo electrónico: mbianchini36@yahoo.com.ar
}

> Resumen - Se presentan dos nidadas atípicas de Tero Común (Vanellus chilensis) en Argentina. Ambas con siete huevos. Las más grandes del país. En una se realiza su seguimiento, brindando la primera evidencia de nidificación de la subespecie Vanellus c. chilensis para la provincia de Neuquén, resultando ser la más austral para Argentina, y constituyendo para la especie nominal la primera evidencia de crías para Sudamérica referido a este tipo de nidadas. La otra, corresponde a la subespecie V. c. lampronotus.

Palabras clave: Biología reproductiva, nidificación, distribución geográfica.

> Abstract - "Atypical Clutch of the Southern Lapwing (Vanellus C. chilensis and V. C. lampronotus, Birds: Charadriidae) in Neuquén and Buenos Aires, Argentina". Two atypical nest of Southern Lapwing (Vanellus chilensis) are presented in Argentina. Both with seven eggs. The largest in the country. One is followed up, providing the first evidence of nesting of the subspecies Vanellus c. chilensis for the province of Neuquén, proving to be the most austral for Argentina, and constituting for the nominal species the first evidence of offspring for South America referred to this type of nest. The other corresponds to the $V$. c. lampronotus subspecies.

Keywords: Reproductive biology, Nesting, Geographic distribution.

El Tero Común es un ave que se distribuye en Sudamérica, desde Panamá hasta el extremo austral de Argentina. Cuenta con cuatro subespecies reconocidas (Del Hoyo, 1996). En Argentina se encuentran tres de ellas (Olrog, 1979; Piersma y Wiersma, 1996; De la Peña, 2016; Salvador, 2016). En la provincia de Neuquén se hallan dos, $V$. c. chilensis (Molina, 1782; Navas y Bó, 1986; Veiga et al., 2005) y V. c. fretensis (Brodkorb, 1934; Navas y Bó, 1986); para la cual recientemente ha sido corroborada su nidificación en dicha provincia (Bianchini, 2017).

Respecto al tamaño de la nidada, esta especie pone normalmente de 3 a 4 huevos (Piersma y Wiersma, 1996; Di Giacomo, 2005; Santos, 2010; De la Peña, 2013),

Recibido: 05/06/17 - Aceptado: 14/11/17 siendo muy raro cinco (Pereyra, 1938; De la Peña, 2013) y más aún un número mayor (De la Peña, 2016). Es escasa la bibliografía existente sobre este tema. Previamente, se han reportado dos casos en Argentina, de nidadas con cinco y seis huevos, en Santa Fe y Buenos Aires respectivamente (Salvador y de la Peña, 2014); y otro caso en Chile, de ocho huevos, en la Región del Biobío (Medrano et al., 2016).

El área de nidificación en Argentina cubre, para $V$. c. chilensis, las provincias de San Juan, Mendoza (Salvador, 2016) y Neuquén (Veiga et al., 2005), aunque sin evidencias publicadas; para V. c. lampronotus, las provincias de Salta, Jujuy, Formosa, Misiones, Chaco, Santiago del Estero, Tucumán, Corrientes, Santa Fe, Córdoba, Entre Ríos, Buenos Aires, San Luis y La Pampa, aunque sin 
datos concretos en estas dos últimas provincias (Salvador, 2016); y para V. c. fretensis, todas las provincias patagónicas (Salvador, 2016), siendo el sur de Neuquén el registro más septentrional (Bianchini, 2017).

\section{NIDADA 1}

El 17 de diciembre de 2016, en intersección de calles Independencia y Arturo Illia (3857'19.93" S - 6802'43.88” O, 264 msnm) de la ciudad de Neuquén capital, departamento Confluencia, provincia de Neuquén, en la ecorregión del Monte de Llanuras y Mesetas, donde son propios el clima templado-árido y escasas precipitaciones (Burkart et al., 1999), se registró un nido de Tero común (Vanellus c. chilensis) con siete huevos, en un predio descampado de 0,4 ha en plena zona urbana y de mucho tránsito (vehicular y ferroviario), uno de sus lados limitado por un desagüe a cielo abierto (Figs. 1 y 2). A partir de allí se realizó un seguimiento de nidificación durante 49 días, hasta el 3 de febrero de 2017. Se llevaron a cabo relevamientos diarios en diferentes horarios, con cámara fotográfica, binoculares, calibre y reflector, realizados a distancia e in situ, éstos últimos en tiempos no superiores a los dos minutos y medio, para evitar provocar molestias a las aves.

Los individuos del caso en estudio corresponden a la subespecie $V$. c. chilensis, ya que se observó la corta longitud y poca densidad

Tabla 1. Características visuales más evidentes y observables en campo, de las tres subespecies de Vanellus chilensis que se encuentran en Argentina.

\begin{tabular}{|c|c|c|c|}
\hline \multirow{2}{*}{ Características } & \multicolumn{3}{|c|}{ Subespecie } \\
\hline & V. c. chilensis & V. c. lampronotus & V. c. fretensis \\
\hline Copete & Corto y grisáceo. & Muy largo y negro. & $\begin{array}{l}\text { Poco visible, corto y grisáceo } \\
\text { (obs. pers.). }\end{array}$ \\
\hline Cabeza y cuello & Tonos gris-celeste, perlado. & Tonos pardo-grisáceo. & Tonos gris-perlado. \\
\hline Parche negro que cubre el pecho & $\begin{array}{l}\text { Más extendido que en } V . c . \\
\text { lampronotus. }\end{array}$ & $\begin{array}{c}\text { Menor que en las demás } \\
\text { subespecies. }\end{array}$ & $\begin{array}{l}\text { Mayor extensión que en las } \\
\text { demás subespecies. }\end{array}$ \\
\hline Alas y cola & $\begin{array}{l}\text { Mayor longitud que las demás } \\
\text { subespecies. }\end{array}$ & $\begin{array}{l}\text { Menor longitud que } \\
\text { V. c. chilensis. }\end{array}$ & $\begin{array}{l}\text { Menor longitud que } \\
\text { V. c. chilensis. }\end{array}$ \\
\hline Aspecto & $\begin{array}{l}\text { Similar a } V . \text { c. fretensis, pero de } \\
\text { mayor tamaño. }\end{array}$ & & $\begin{array}{c}\text { Similar a V. c. chilensis, pero } \\
\text { claramente de menor tamaño, mas } \\
\text { notorio en los tarsos. }\end{array}$ \\
\hline
\end{tabular}

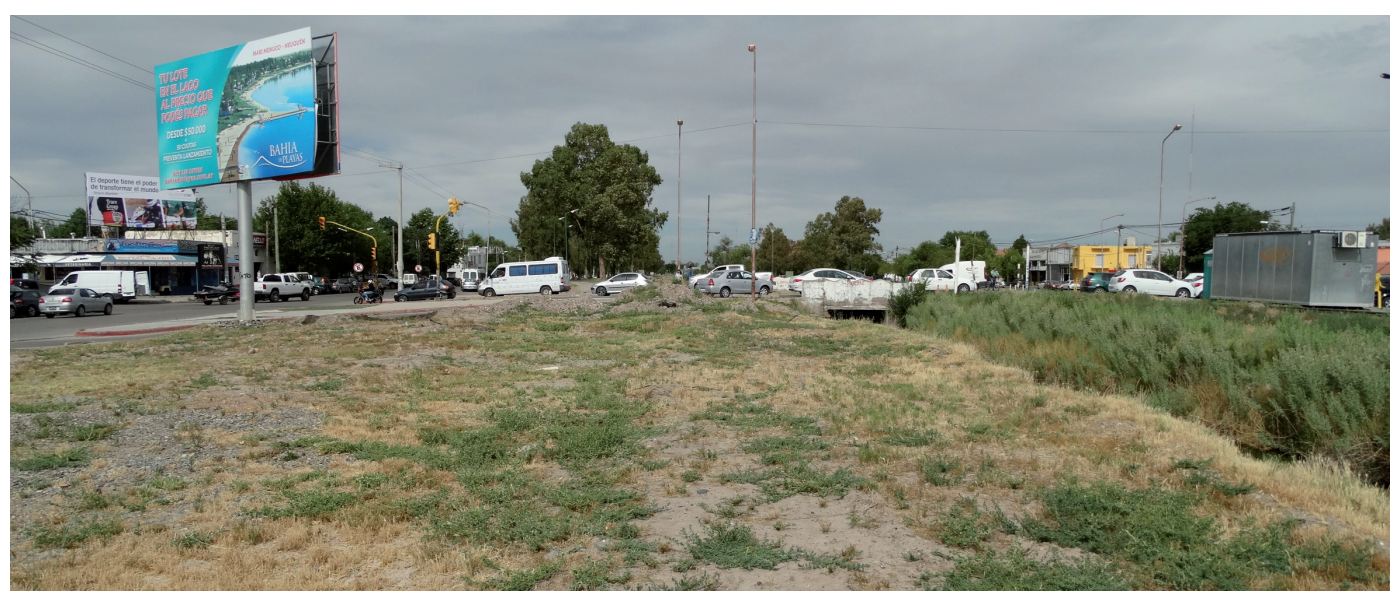

Figura 1. Panorámica del predio donde se halló el nido de Tero Común (V. C. chilensis) con siete huevos. Intersección calles Independencia y A. Illia. Ciudad de Neuquén cap., dpto. Confluencia, provincia de Neuquén, Argentina. Foto: M Bianchini. 


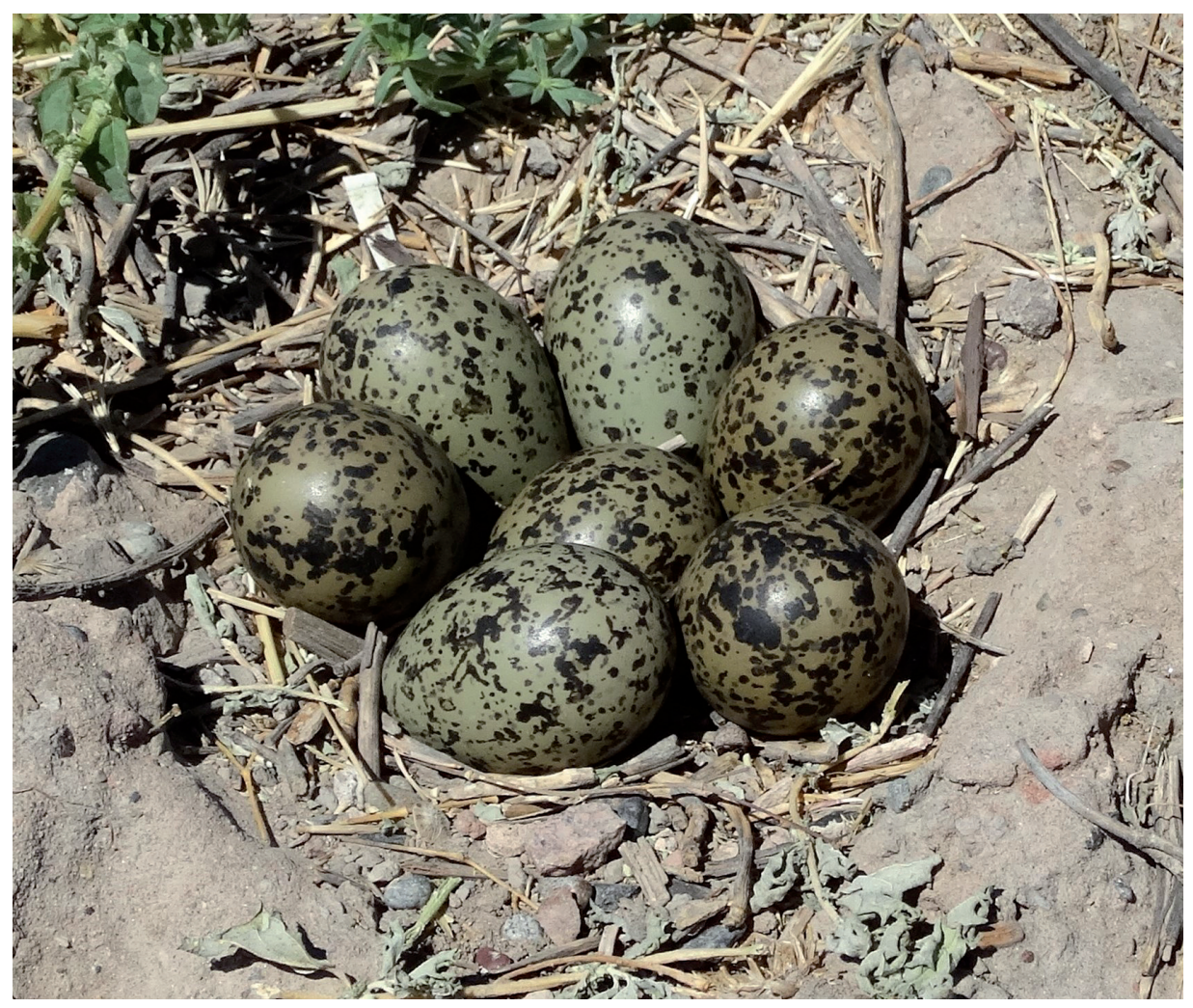

Figura 2. Nidada atípica de Tero Común (V. c. chilensis) con siete huevos. Ciudad de Neuquén cap., dpto. Confluencia, provincia de Neuquén, Argentina. 18 de diciembre de 2016. Foto: M. Bianchini. Nidada conformada por la puesta de dos hembras. Nótese las dos tonalidades entre las dos posturas, una constituída por el huevo del centro, los dos a la derecha de éste, y el de la izquierda, más amarronada, en contraste al verde claro de los tres restantes de la otra.

de plumas del copete, que es grisáceo, y no negro ni tan evidentemente largo (Figuras 3 , 4 y 5 ; Tabla 1 ); la cabeza y el cuello presentaban tonos gris-celeste y no pardo-grisáceos; y el negro del pecho era más extendido, en contraste a V. c. lampronotus. Otras diferencias las constituyen el tamaño y los tarsos, que no son tan chicos y tan cortos respectivamente; ni el negro del pecho es tan extendido, como los de $V$. c. fretensis (Figuras 3, 4 y 5; Tabla 1). Por otro lado, se destaca que se hallaron en una zona considerada no propia de las últimas subespecies citadas; y que se ha reportado que en el área geográfica de nidificación no habría superposición entre ellas (Molina, 1782; Wagler, 1827; Brodkorb, 1934, Navas y Bó, 1986; Piersma y Wiersma, 1996; Salvador, 2016; Bianchini, 2017).
Desde el inicio del seguimiento tres ejemplares adultos estuvieron al cuidado del nido (Fig. 3) como generalmente ocurre en esta especie (Walters y Walters, 1980; S. Salvador com. pers.). Dicho cuidado era muy agresivo ante intrusos, realizando previamente parada de falso nido y luego reiterados ataques en vuelo rasantes con voces estridentes (Hudson, 1974). El 29 de diciembre de 2016, se halló un pichón con pocas horas de eclosionado y cinco huevos, habiendo el sexto huevo desaparecido (Fig. 6). En este día el autor recibió ataques directos con los espolones y defecaciones de las aves. Al día siguiente y hasta el 24 enero de 2017 permanecieron en el nido sólo los cinco huevos, mientras que el pichón había desaparecido. A fines de diciembre se notó un decrecimiento del cuidado defensivo y agresivo ante intrusos por 


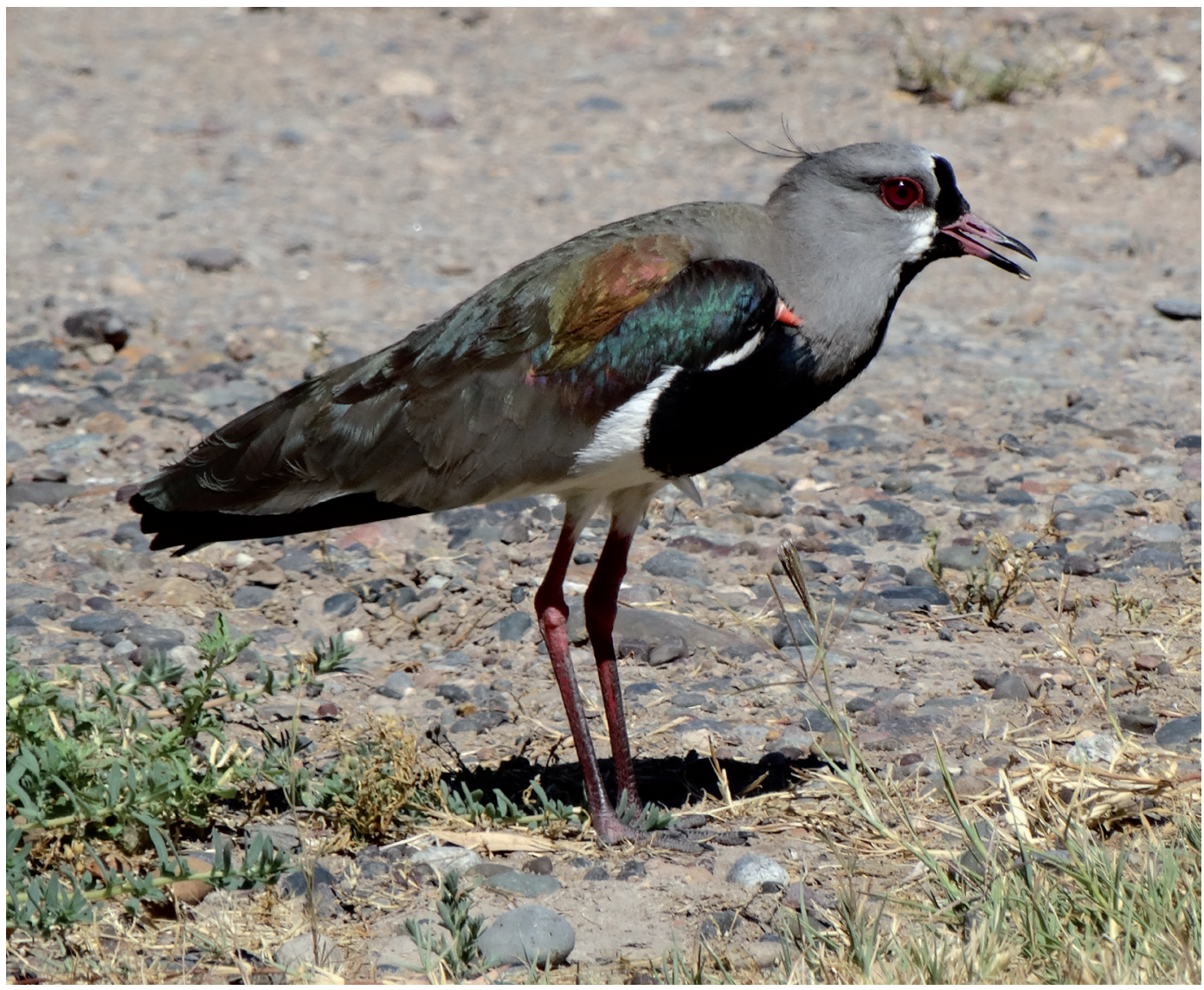

Figura 3. Uno de los tres adultos de Tero Común (V. c. chilensis) del nido con siete huevos. Ciudad de Neuquén cap., dpto. Confluencia, provincia de Neuquén, Argentina. 26 de diciembre de 2016. Foto: M Bianchini.

parte de los adultos, aunque éstos permanecían en los alrededores. En la madrugada del 25 de enero de 2017 se encontró en el cauce del desagüe, metidos en el agua somera, a dos pichones o juveniles, de 27 y 28 días de edad (Fig. 7a) acompañados por uno de los ejemplares adultos, a unos $10 \mathrm{~m}$ de los cinco huevos de la nidada, que parecían haber sido abandonados. Desde entonces se continuó con el monitoreo también por las noches, donde sólo en horario nocturno se dejaban ver las crías por unos instantes, antes de esconderse en la vegetación arbustiva del desagüe, siempre acompañados por un solo adulto que cuidaba de las crías. A veces también se halló a los pichones dormitando parados, entre el plumaje de la zona del pecho y ventral del adulto. Al detectar la pre- sencia del observador, el adulto, emitía voces de alarma a los otros dos adultos, alejados al menos unos $100 \mathrm{~m}$ de distancia, los cuales acudían sin demoras y con voces de alarma $\mathrm{y}$ actitud defensiva.

El 27 y 29 de enero de 2017, durante la media noche, se encontró a un adulto, pero con una sola cría de un mes de vida, en el cauce del desagüe (Fig. 7b). En cada una de estas noches se detectó a un gato doméstico (Felis catus) escondido entre los arbustos acechando desde la orilla del desagüe a muy pocos metros de los teros. El 31 de enero de 2017, en todo el día e inclusive a la noche, se observó a un adulto pero sin ninguna cría. Y desde el 1 de febrero de 2017 ni los adultos ni las crías fueron encontrados en el predio. Sólo a los primeros, uno a unos 100 


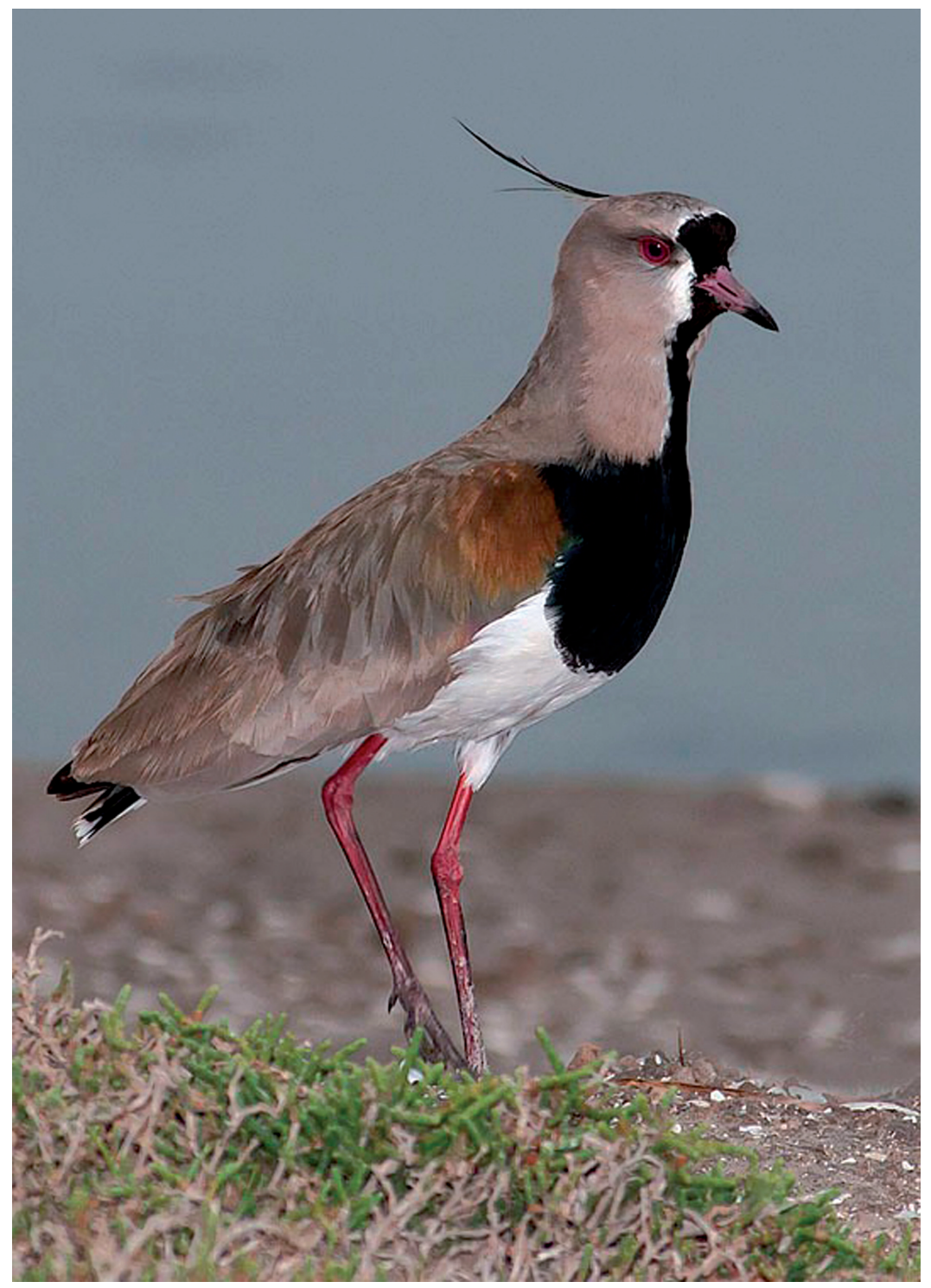

Figura 4. Ejemplar de V. c. lampronotus. Mar Chiquita, partido homónimo, provincia de Buenos Aires, Argentina. 9 de marzo de 2011. Foto: E Polverini.

$\mathrm{m}$ y a los otros dos a unos $300 \mathrm{~m}$, alejados del predio. Esto mismo sucedió en los días posteriores, sin visualizar ninguna cría en los alrededores.

A partir de lo relevado, el éxito de eclosión fue de 28,56 \% (dos de siete huevos), y al final del seguimiento, si bien las crías alcanzaron el mes de vida, desaparecieron, sin lograr concluir el éxito de crianza. El área de cría fue notoriamente menor, entre 3 a 7 veces en contraste a las áreas mínimas esti- madas 1,33 a 2,8 ha indicadas en Gallegos Luque (1984).

El registro presentado brinda para la especie nominal (Vanellus chilensis) la primera evidencia de cría de nidadas atípicas en Sudamérica. También resulta ser la primera evidencia publicada de nidificación de $V$. $c$. chilensis para la provincia de Neuquén, y la más austral de dicha subespecie para el país, a unos $380 \mathrm{~km}$ al sur de la Reserva Provincial Laguna Llancanello (Sosa, 1995). 


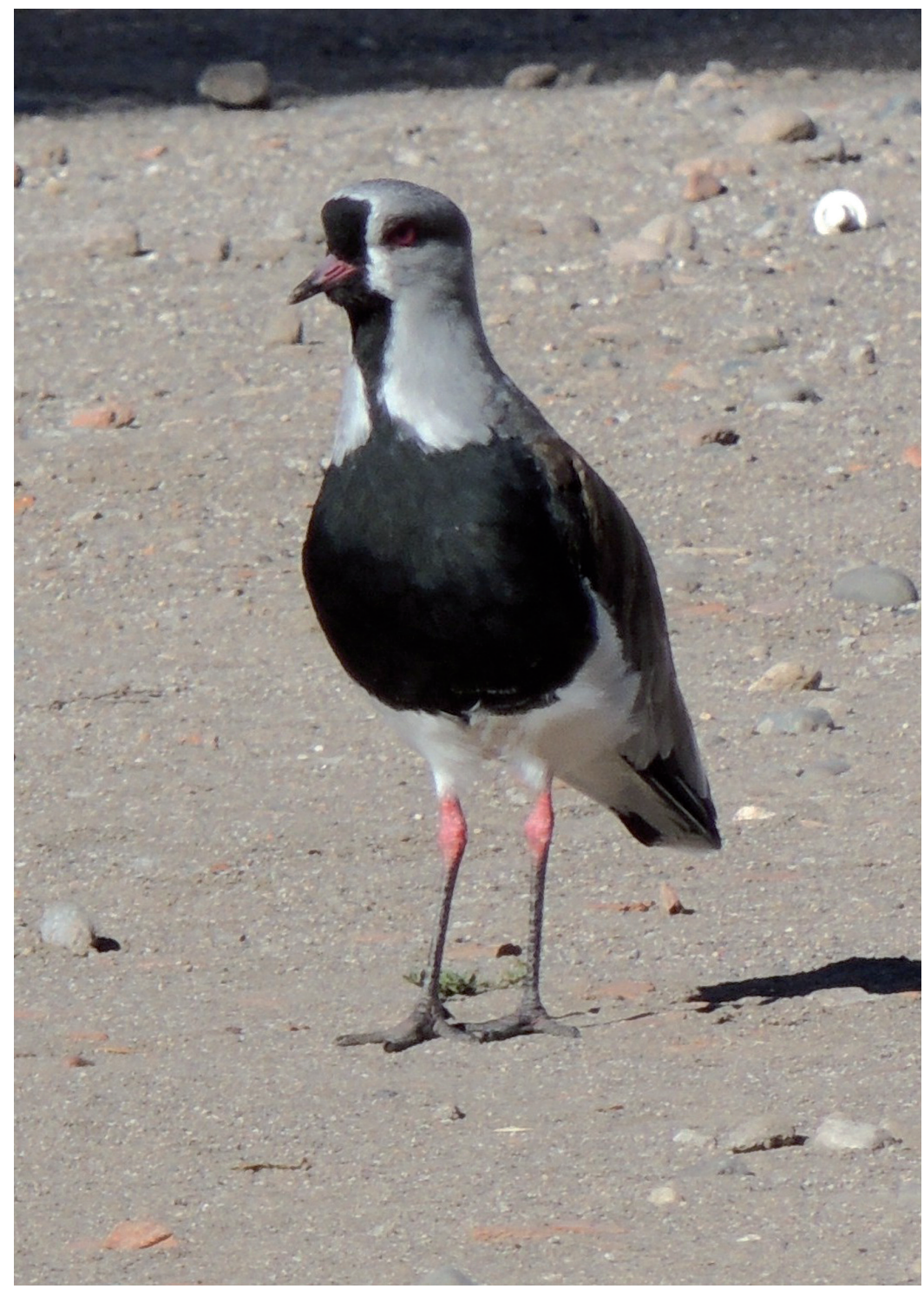

Figura 5. Ejemplar de V. c. fretensis. Los Antiguos, dpto. Lago Buenos Aires, provincia de Santa Cruz, Argentina. 20 de enero de 2015. Foto: M A Villarruel.

Las medidas de los huevos fueron (eje mayor por eje menor) (mm): N1: 48,6 x 34; N2: 49 x 34; N3: 46,4 x 34,5; N4: 49,2 x 33; N5: 49 $\mathrm{x} 33,5$; media $=48,44 \times 33,8(\mathrm{~N}=5)$.

Dado el tamaño de la nidada, surge la incertidumbre si la postura fue a cargo de una o más hembras, debido a que en el lugar estuvieron siempre presentes y de guardia constante tres adultos. Por tanto uno de ellos podría haber estado oficiando, en trío, como ayudante en la cría. Sin embargo al observar las fotografías se visualizan dos tonalidades diferentes en los siete huevos de la nidada, donde el huevo del centro, los dos a la derecha de éste, y el de la izquierda poseen una tonalidad más amarronada en contraste al verde claro de los tres restantes (Fig. 2), dando la posibilidad que se trate de una nidada conformada por la postura de dos hembras, tal lo estudiado en Saracura et al. (2008). Vale notar que cada postura de la nidada 1 no superó los cuatro huevos. 


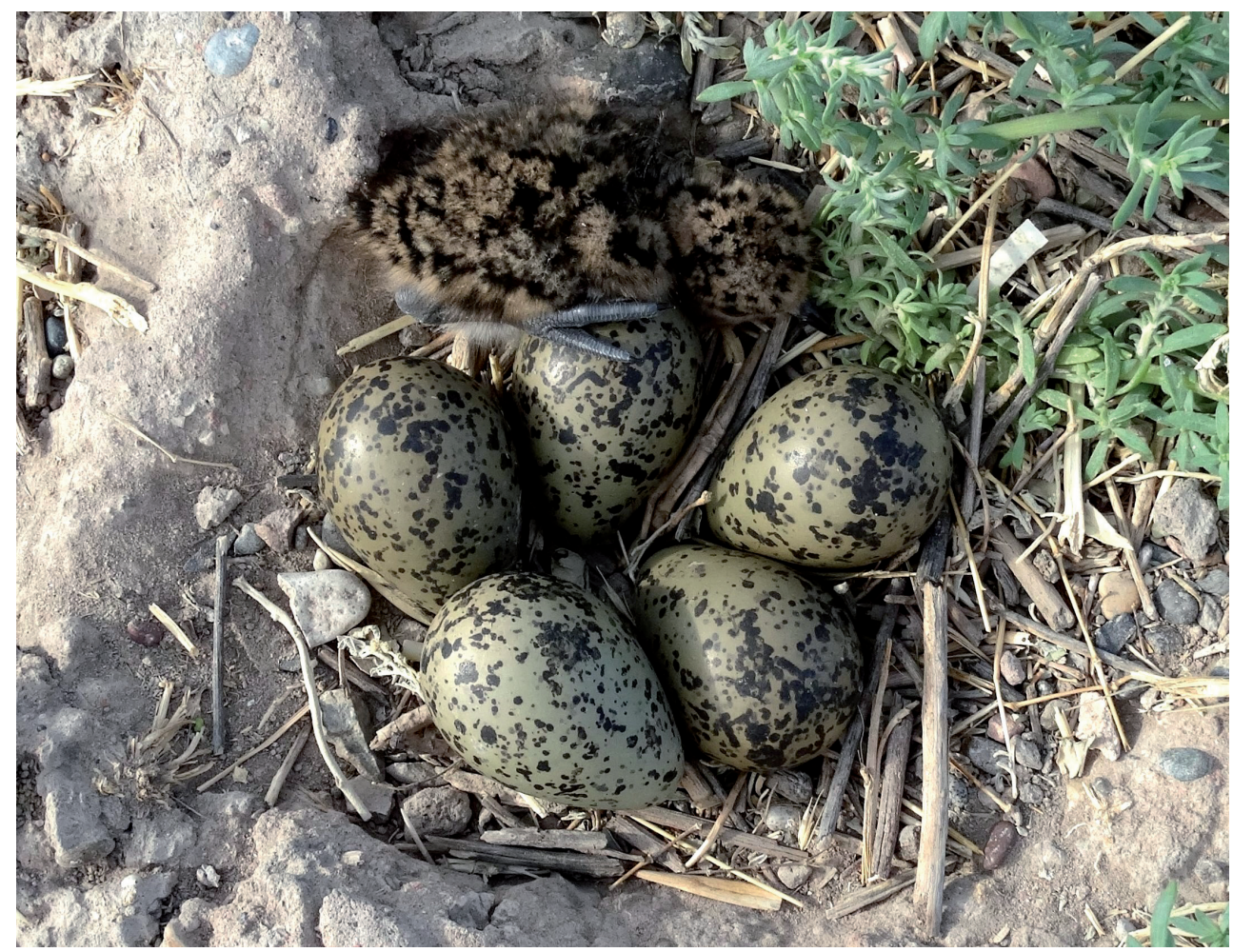

Figura 6. Pichón de Tero Común [ $V$. c. chilensis] con pocas horas de vida. Ciudad de Neuquén cap., dpto. Confluencia, provincia de Neuquén, Argentina. 29 de diciembre de 2016. Foto: M Bianchini.

\section{NIDADA 2}

El 24 de octubre de 2015, en San Pedro (3703'40.64" S - 5705'35.03” O, 4 msnm), partido de Madariaga, provincia de Buenos Aires, en la ecorregión de Pampa/Pradera Pampeana. En ese sitio el clima es templadohúmedo a subhúmedo, con veranos cálidos y precipitaciones anuales que varían entre 600 a 1100 mm (Burkart et al., 1999), Rocío Aluminé Sangorrín registró un nido con siete huevos, en zona rural (Fig.8; R. A. Sangorrín com. pers. 2017). Esta nidada correspondería a la subespecie $V$. c. lampronotus, por su ubicación geográfica, fuera del rango reproductivo de las otras dos subespecies. Las Nidadas 1 y 2 resultan ser las más grandes reportadas para Argentina.

La existencia de dos tonalidades diferentes en los huevos de un mismo nido (postura de dos hembras), se apreció también en la nidada 2 , con la distinción que una cuenta con cinco huevos, por lo que se estaría tratando de un caso de máxima postura efectuada por una hembra. En los casos de Salvador y de la Peña (2014) y Medrano et al. (2016) también se aprecia variación en el patrón de coloración de los huevos. Además de las diferencias cromáticas recién mencionadas, en todos los casos aludidos de nidadas atípicas, se observa la repetición de un patrón de tonalidad: los huevos de $V$. $c$. chilensis resultan ligeramente más oscuros que los de $V$. c. lampronotus.

\section{AGRADECIMIENTOS}

A Cynthia Arenas y a Raúl Navarro por avisar la existencia del nido de Neuquén. A Rocío Aluminé Sangorrín, Edith Polverini y Miguel Angel Villaroel, por brindar sus registros. A Sergio Salvador por facilitar bibliografía y revisar la versión inicial del manuscrito. 

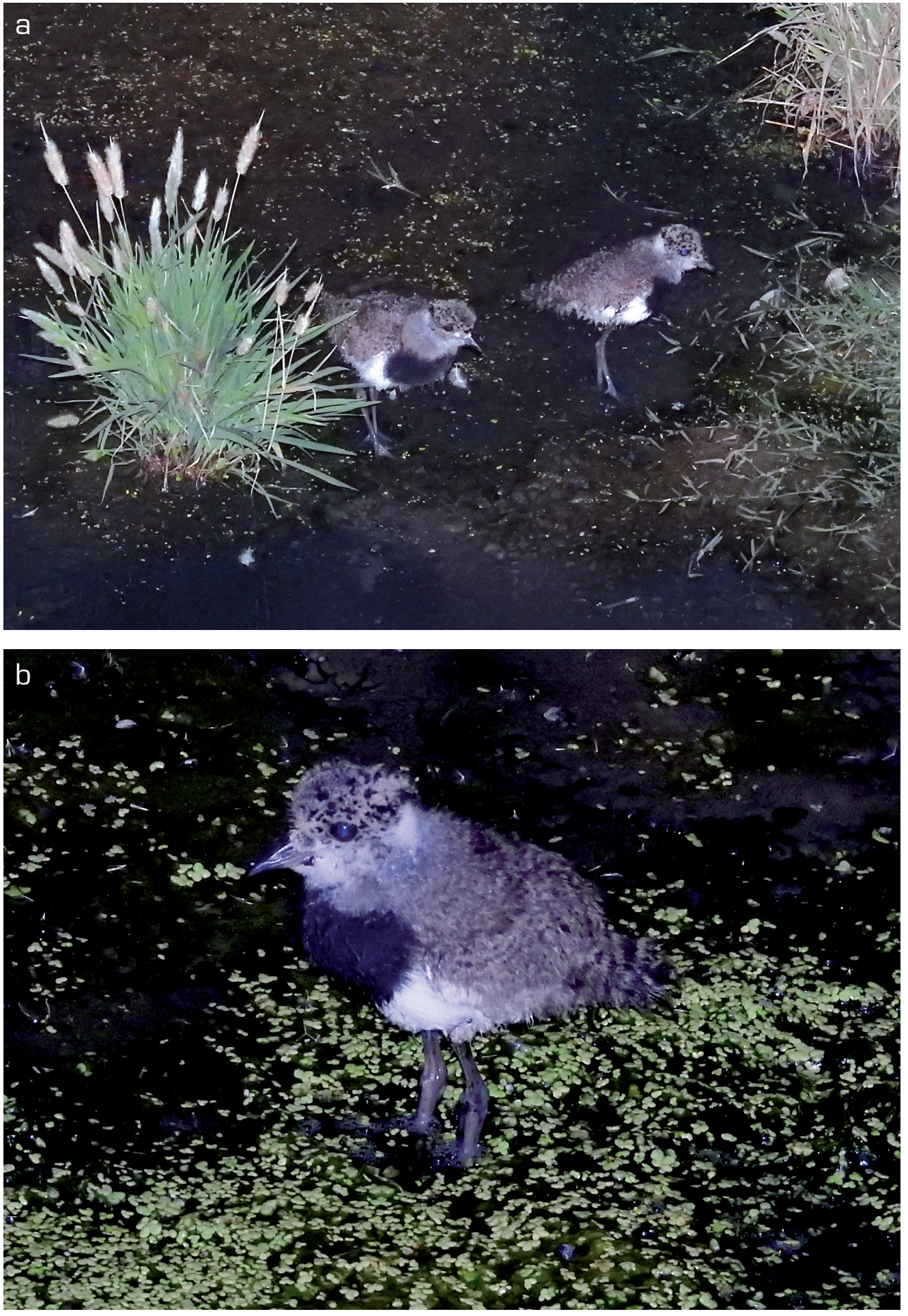

Figura 7. Cría de nidada atípica para la especie nominal (Vanellus chilensis). Ciudad de Neuquén cap., dpto. Confluencia, provincia de Neuquén. Argentina: a) Juveniles de 27 y 28 días de vida. 25 de enero de 2017; b) Juvenil de un mes de vida. 27 de enero de 2017 . Fotos: M Bianchini. 


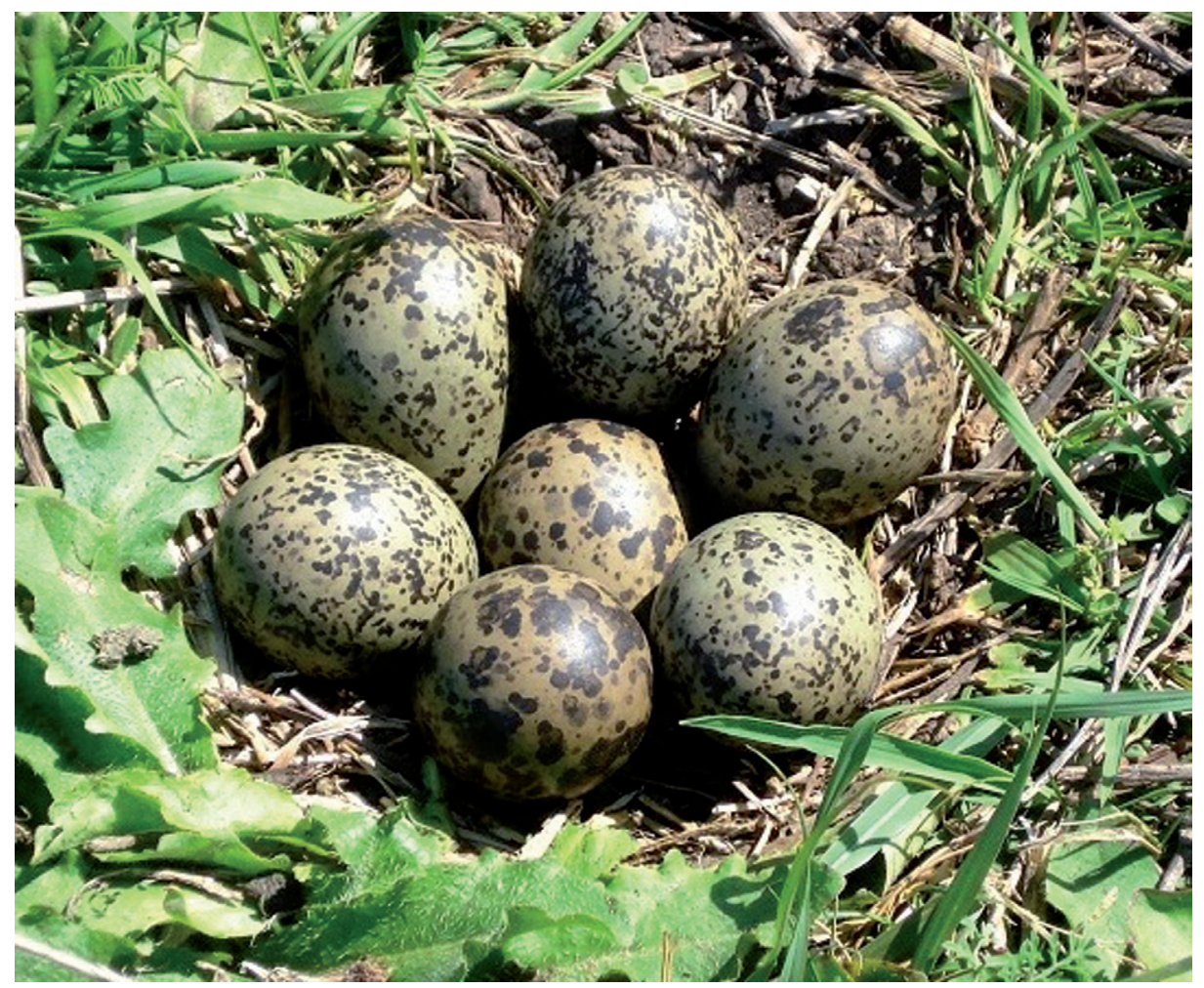

Figura 8. Nidada atípica de Tero Común (V. c. lampronotus) con siete huevos. San Pedro, partido de Madariaga, provincia de Buenos aires, Argentina. 24 de octubre de 2015. Foto: R A Sangorrín. Nidada conformada por la puesta de dos hembras. Nótese las dos tonalidades entre las dos posturas, una constituída por dos huevos, el del centro y el de abajo (la más amarronada), y la otra por los cinco huevos restantes.

\section{LITERATURA CITADA}

Bianchini M. 2017. Contribuciones al conocimiento de la nidificación de aves en la Patagonia, Argentina. Historia Natural (Tercera serie), 7 (1): 69-83.

Burkart R., Bárbaro N.0., Sánchez R.0., Gómez D.A. 1999. Ecorregiones de la Argentina. Administración de Parques Nacionales, PRODIA, 1-43.

De la Peña M.R. 2013. Nidos y Reproducción de las aves argentinas. Serie Naturaleza, Conservación y Sociedad № 8. Ediciones Biológica. Santa Fe. 594 pp.

De la Peña M.R. 2016. Aves Argentina: descripción, comportamiento, reproducción y distribución. Charadriidae a Trochilidae. Comunicaciones del Museo Provincial de Ciencias Naturales "Florentino Ameghino" (Nueva Serie) 20 (1): 1-627.

Del Hoyo, J., Elliott, A. Sargatal, J. 1996. Handbook of the Birds of the World. Volume 3: Hoatzin to Auks. Lynx Edicions. Barcelona. $821 \mathrm{pp}$.
Di Giacomo A.G. 2005. Aves de la Reserva El Bagual. Pp. 203-465, en Di Giacomo A.G. y S.F. Krapovickas [eds]. Historia natural y paisaje de la Reserva El Bagual, provincia de Formosa, Argentina. Inventario de la fauna de vertebrados y de la flora vascular de un área del Chaco Húmedo. Temas de Naturaleza y Conservación 4. Aves Argentinas/AOP, Buenos Aires.

Gallegos Luque D. 1984. Aspectos de la biología reproductiva del Tero Común Vanellus chilensis (Gmelin). I: Comportamiento y territorialidad. Hornero, 12 : 150-155.

Hudson G.E. 1974. Aves del Plata. Libros de Hispanoamérica. Buenos Aires. 361 pp.

Medrano F., Cerpa P., Castro-Pastene C., Gutiérrez Guzmán H. 2016. Tamaño de puesta inusualmente grande para el queltehue (Vanellus chilensis) en el centro-sur de chile. Boletín Chileno de Ornitología, 22: 210-211. 
Navas J. R., Bó N. A.1986. Revisión de las subespecies argentinas de Vanellus chilensis (Aves, Charadriidae). Neotrópica, 32: 157-165.

Olrog C.C. 1979. Nueva lista de la avifauna argentina. Opera Lilloana (XXVII). Tucumán. 324 pp.

Pereyra J.A. 1938. Aves de la zona ribereña nordeste de la provincia de Buenos Aires. Memorias del Jardín Zoológico de La Plata, 9: 1- 304.

Piersma, T., Wiersma P. 1996. Family Charadriidae (Plovers). Pp. 384-442, en del Hoyo, J., Elliot, A. y Sargatal, J. (eds.). Handbook of the Birds of the Word. Vol. 3. Hoatzin to Auks. Lynx Edicions, Barcelona.

Salvador S.A., De la Peña M.R. 2014. Nidadas anormales de Tero común (Vanellus chilensis] en Argentina. Biológica, 17: 60-61.

Salvador S.A. 2016. Distribución Reproductiva de las Aves de Argentina y sus Territorios. Informe inédito. Córdoba, Argentina. $333 \mathrm{pp}$.
Santos E.S.A. 2010. Southern Lapwing (Vanellus chilensis), Neotropical Birds Online [T. S. Schulenberg, Editor]. Ithaca: Cornell Lab of Ornithology; retrieved from Neotropical Birds Online: http://neotropical.birds.cornell.edu /portal/species/overview?p_p_spp $=144596$

Saracura V., Macedo R., Blomqvist D. 2008. Genetic parentage and variable social structure in breeding southern lapwings. Condor, 110: 554-558.

Sosa H. 1995. Actualización de la lista de avifauna de la Reserva Provincial Laguna Llancanelo, Malargüe, Mendoza. Presencia estacional, referencias de hábitat y nidificación. Multequina, 4: 65-75.

Veiga J., Filiberto F., Babarskas M., Savigny C. 2005. Aves de la provincia de Neuquén. Patagonia Argentina. Lista Comentada y Distribución. RyC Editora, $184 \mathrm{pp}$.

Walters J., Walters B. 1980. Co-operative breeding by Southern lapwings Vanellus chilensis. Ibis, 122: 505-509. 